\title{
PLACEMAKING AS AN APPROACH TO FOSTER CULTURAL TOURISM IN HERITAGE SITES
}

\author{
HAITHAM SAMIR, SALWA SAMARGANDI \& MOHAMMED F. M. MOHAMMED* \\ Department of Architecture, Effat University, Kingdom of Saudi Arabia
}

\begin{abstract}
The accelerating competition among cities brought on by globalization has resulted in a significant concern to harness cultural assets heritage recovery as a form of placemaking to establish relative competitive advantage and consequently attract more visitors and cultural tourism. It is evident that the physical heritage assets and cultural expressions play a substantial part in establishing a sense of place. Other factors related to the needs of tourists and visitors affect the degree of attractiveness and foster greater attachment to the place. In that sense, developing such heritage sites requires a comprehensive approach that considers various aspects in both tangible "physical" and intangible "unphysical" factors related to those spaces in which it creates authenticity and sense of place. This research attempts to explore the notion of placemaking as an approach for developing and conserving heritage sites, hence, promoting successful cultural tourism that balances the development between people and place. The research will consider using a qualitative approach, supported by statistical analysis through conducting a structured questionnaire for experts in the field in order to verify and weighing the importance of proposed qualities that contribute to building the suggested model. The research will result in demonstrating the proposed model of placemaking that is specialized for heritage sites, which it aims to achieve prosperous cultural tourism.
\end{abstract}

Keywords: heritage sites, context conservation, urban historic landscape, placemaking, cultural tourism, tangible, intangible, placemaking model.

\section{INTRODUCTION}

The significance of heritage and cultural sites has been enlightened by the public than times before, especially through the transformation of notions in planning policies which many countries are going through [1]. Therefore, understanding urban heritage conservation and development approaches are essential, by considering the embedded dimensions forming heritage whether its tangible or intangible which it holds values, emotions and stories [2], [3]. This was acknowledged and considered by recent approaches concerning heritage development where they called "historic urban landscape" which is an approach that reflects a holistic image into urban conservation including the entire urban context [4]. This research aims to investigate the subject deeply and try to configure an adapted approach that considers the three main factors which are people, place, and tourism, where this approach aims to achieve successful cultural tourism in order to benefit the country which is the main goal after all. Placemaking is an approach which focuses on the relationship between man and the place as the recent approaches of heritage recommends. Not only this but also it focuses on the values concerned about places to strength the soulful aspect and authenticity [5].

Since this subject is explicitly always aligned with heritage and cultural tourism, one must consider this while development to make it successful, humane, and pleasant for people to experience and avoid damages that could occur to heritage [6]. This research will investigate the notion of placemaking in theory and practice, its development through years. In addition to its application on real projects, where later the research will try to identify the relationship

\footnotetext{
* ORCID: http://orcid.org/0000-0003-0119-5033
} 
between the primary factors and adapted to the model by developing a tailored approach resulting from this research.

\section{CHANGING PARADIGMS FOR INTERVENING IN URBAN HERITAGE PLACES}

Today, urban heritage sites need attention and development as any other urban areas. It is essential to have an identified vision with a clear purpose and direction that forms a comprehensive place-making. According to division, there is a low chance for urban places to succeed without a comprehensive development that allows it to grow and sustain [7]. Where such places especially sensitive areas such as heritage and cultural places, require a clear vision to deal with it to maintain protection and preservation of existed assets and qualities and identify maximum and future potentials. Therefore, understanding a holistic framework and process that integrates all aspects that contribute to shaping the place is highly demanded to ensure its success. The placemaking approach was initiated by several pioneers such as Jacob and William Whyte, where they promoted humans as the center of development. This made this theory unique as it combines the essential factors which current urban life needs. Developing cultural and heritage places and promoting tourism need high consideration of factors that could keep those place thriving and lively, therefore placemaking where ideal to be considered. However, the approach remained generic through its development for years in both theory and practice. The research contributes new perspective on dealing with urban heritage sites by building on the placemaking approach which counts for the connection between people and the place. The goal is to allow for enhanced experiences with tangible and intangible forms of heritage and support relations with physical, social, and economic settings of the place.

\section{THE KEY ATTRIBUTES OF PLACEMAKING FOR TOURISM IN HERITAGE SITES}

If urban heritage sites are to attract people for different purposes, their public realm should be planned and designed in a manner that fosters its sense of place and identity. Consequently, turning into a place where people like to stay, work, and visit. The public realm including the quality of streets, plazas, open spaces..., etc. Is playing a significant role in the determination of the competitiveness of the place among others. Heritage sites are not only acknowledged by the quality of its composite physical elements, but also by other attributes related to human values, social activities, intangible factors, and people satisfaction. Thus, the determination of various attributes that make the place and identify its quality is essential to determine the correct interventions and actions that lead to the prosperous future of heritage sites.

\subsection{Access and linkage attribute}

Access and linkage attribute is concerned about the movement network from and into the places which study the overview layout and intersection of public streets, roads, and paths. Where it examines the interrelation system of streets that integrates pedestrian and bike lanes, public transport, and other vehicle facilities. It aims to provide reachable destinations for people to access places and activities easily. Additionally, to provide spaces for services such as infrastructure utilities and parking spaces. Ensuring the movement network functionality by designing to be legible, convenient, and safe and services for users can achieve excellent walking and movement activity. 
Its importance lies in providing equitable access to places, facilities, and public transport, also, its effect on people's mobility and safety. This results from well-studied connections to places and linkages to different modes of transportation. Streets play an essential role in developing the public realm, where it considers part of public spaces that support social interaction and places for cultural expression [8]. The following are the main aspects that concern access and linkage domain.

- Continuity

- Proximity

- Connected
- Readable

- Convenient

- Accessible
- Walkable

\subsection{Comfort and image attribute}

The comfort and image attribute is concerned with the aspects that contribute to the visual preferences and human needs and satisfaction. It plays a vital role in connecting the man with the place and its liveability. These aspects are translated through both tangible and intangible characteristics that interrelate with each other. According to Svirplys [9], the image of the place is formed from a set of attributes that feature in and out. The image is defined as an impression of a specific place translated through a mix of induvial, socio-cultural, and physical aspects of a place, which gives it spatial and non-spatial form, for example, sense of place. On the other hand, comfort which is emanated from the physical setting of the place. In which it refers to the human experience and feeling within a place where it reflects an engaging, informative and formative journey. These aspects could be listed as follows:

- Safe

- Visually pleasant

- The sense of place

- Thermal comfort
- Clean

- Historic

- Satisfactory
- Sittable

- Local identity

- Enjoyable

3.3 Uses and activity attribute [10], [11]

- Active

- Unique

- Celebratory
- Fun

- Useful

- Sustainable
- Vital

- Indigenous

- Experienced

\subsection{Sociability attribute [11]}

- Divers

- Pride

- Celebratory
- Stewardship

- Interactive

- Sustainable
- Cooperative

- Welcoming

- Experienced

\subsection{Context and conservation attribute}

By adapting the place making model for urban heritage sites, there must be aspects that are concerned with such sensitive urban sites. Therefore, the researcher suggested this domain added to the framework of placemaking in which is concerned with the urban context and conservation. Urban heritage sites have a valuable urban context different than any others where it holds plenty of stories, history, and meanings. The urban context consists of the cultural, social-economic moreover, visual, and physical setting. A successful relationship between those components with contexts could result not only in well-functioned places but also in a more significant visual appearance. This would contribute to forming more of a 
meaningful experience, hence, achieving a sense of place. According to Planning Policy Division, it is described by understanding place [7]. It is essential to understand the site and its context to form any proposal [13]:

- Contextually integrated

- Protected
- Preserved

- Valuable
- Maintained

- Aesthetically appealing

\subsection{Site interpretation}

There are some unique features considered to be important in heritage and cultural contexts since they hold various stories and valuable history for a place to tell. Through design and flourishment, we communicate heritage. As described by scholars in the literature that through proper interpretation, the history of a place and its people can come along also to engage residents and tourists alike. Interpretative tools must be clear, specific, and wellstudied to achieve the right message for people. The aim is to increase people's interest in the place and ignite their imagination to encourage them to interact with the places. Hence, a good reputation can be achieved. Creativity in promoting, storytelling and activities of the place is essential rather than traditional ones [12].

- Site interpretation

- Soulful
- Human-oriented

- Memorable
- Authentic

- Informative

According to previous definitions, it is clear that each aspect can be measured through multiple indicators, where, in many cases, the indicators have an impact on many aspects from different domains. This shows the need for a comprehensive framework model that can demonstrate the relationships between the various qualities and their indicators.

\section{PLACEMAKING PROPOSED MODEL FOR HERITAGE SITES}

This research aims to build an integral placemaking model for heritage sites in which it encompasses holistic aspects that is concerned with these sensitive places, essential urban design components, and creative aspects which support cultural tourism. In that sense, proposed indicators have also been taken into consideration to assess the achievement level within the design process and outcomes. Additionally, the proposed model also aims to be used by practitioners in the field. Decision-makers is a bulleted list.

\subsection{Objectives of the model}

The proposed placemaking model in heritage sites aims to create a practical tool to be used by professionals, in different levels:

- To provide the right guidance for urban planners and urban designers, while the design process by considering the critical aspects.

- To define a holistic criterion for evaluating proposed projects.

- To provide clear guidance for decision-makers to take actions according to priority and importance.

\subsection{Methodology and process of building the model}

Building an adapted model for that specific type of places requires a full understanding of placemaking approach to identify the intersection among them in which it contributes to the original vision. Therefore, the researchers have developed a generic taxonomy of 
placemaking approaches to configure that initial base of forming the model referring to its aim and objectives. A hybrid approach of strategic and creative placemaking was considered while developing the new model, where strategy is concerned with developing an entire area focusing on the economic growth aspect and people satisfaction while creative is an activitybased approach which involved with cultural and social vibrancy among a place. On the other hand, the generic placemaking for successful public realm model which is more related to the strategic approach but on a broader perspective and more of human-oriented. Together, one can establish a definite foundation that covers a holistic border of understanding placemaking.

Although the proposed classification has identified more than five strategies based on defined taxonomy, there is a count of alternative approaches to generate an adaptive placemaking model strategy. This integration could lead to an effective practical approach to developing a customized one which can perform adequately in a place. Considering the characteristic of each relevant approach; a potential hybrid strategy has been defined. Where embedded factors like activities, arts, and creativity can be integrated with other approaches. This base helped the researchers to identify and select the foundation of structuring the model to bridge between theory and practice. The researchers adapted the recent developed generic placemaking model for the prosperous public realm. It is concerned of humanized places considering tangible and intangible aspects within the place as well as it holds holistic characteristics for developing an urban area oriented for people, which is a crucial factor the researchers aimed to consider in building the adapted model of placemaking in heritage sites in order to ensure a quality developed places not only cultural (Fig. 1).

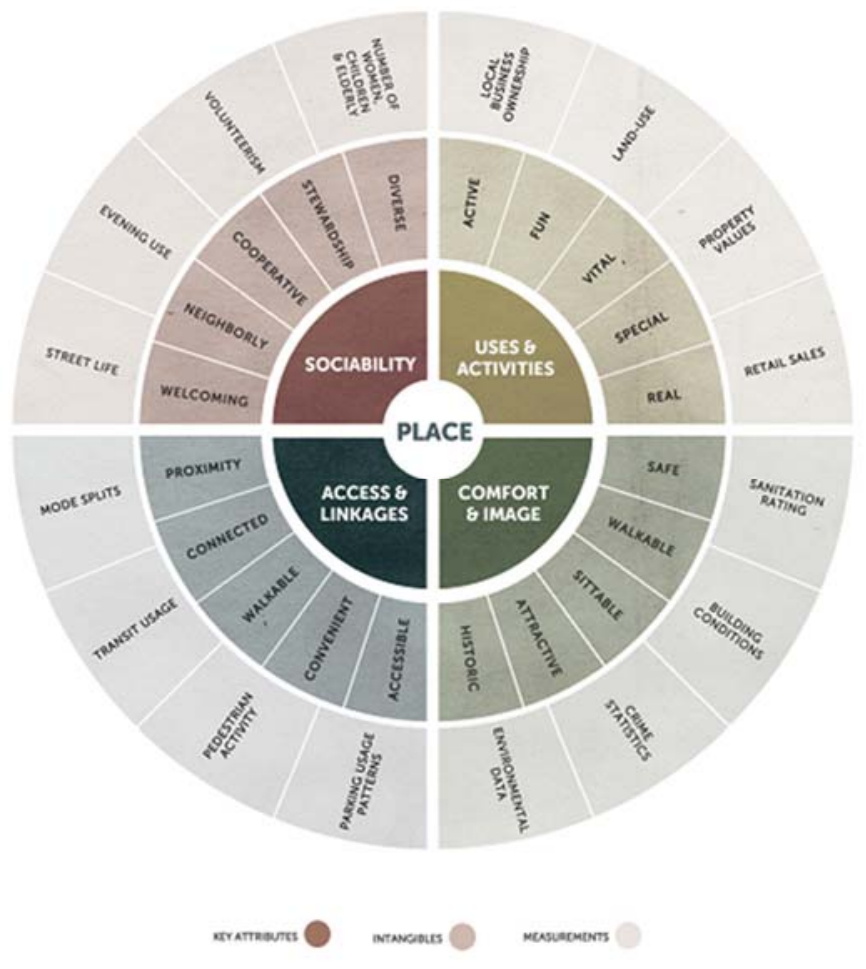

Figure 1: The generic placemaking model developed by PPS [14]. 
The placemaking model, introduced by Project for Public Spaces Inc. [14], encompasses generic key attributes that should be fulfilled to achieve a prosperous urban place regardless of the variation that could occur from a place to another. In that sense, one should understand the type of urban area that wanted to be developed in order to identify the aspects that fit well in every particular area. In this research, the four significant domains, including their aspects, will be adapted and explored, representing the aim of achieving successful heritage site development for cultural tourism. Consequently, this requires another layer of modifications by adding particular domains and aspects that are concerned with heritage sites, which are the subject of this research, along with the aspects that support creativity and cultural tourism activities, which refers to thoughtful approaches mentioned earlier. Along with that, the attempt model will also propose additional indicators which impacts the achievement of those aspects in several dimensions.

\subsection{Model adaptation}

Following the modifications, we found that some of the listed aspects are irrelevant. Concerning heritage and cultural sites require specific qualities that contribute to preserve, protect, and conserve heritage assets value by all means physically and unphysically. Therefore, the researchers added two main domains (attributes) to the original one extracted from the study of heritage conservation modern approaches and cultural tourism, where they are significant factors contribute to assessing the developments in the assigned context. These domains are named as context and conservation which is concerned with the physical setting of the heritage that consists of preserved, protected, valuable, aesthetics and urban context integration. While the other is named site interpretation as it refers to the full range of potential activities intended to raise public awareness and strengthen understanding of the complexities of the heritage sites [15]. In addition to that, some aspects were replaced the avoided ones in comfort and image domain by adding, enjoyable, satisfactory, local identity, sense of place and thermal comfort. Also, in the activities domain, the aspect (experienced) has been added contributing to the significant activities within the context and the touristic movements. Fig. 2 showcases an initial phase of forming the model, where it shows the added two domains along with the original four domains as well as the added aspects under each domain (attribute).

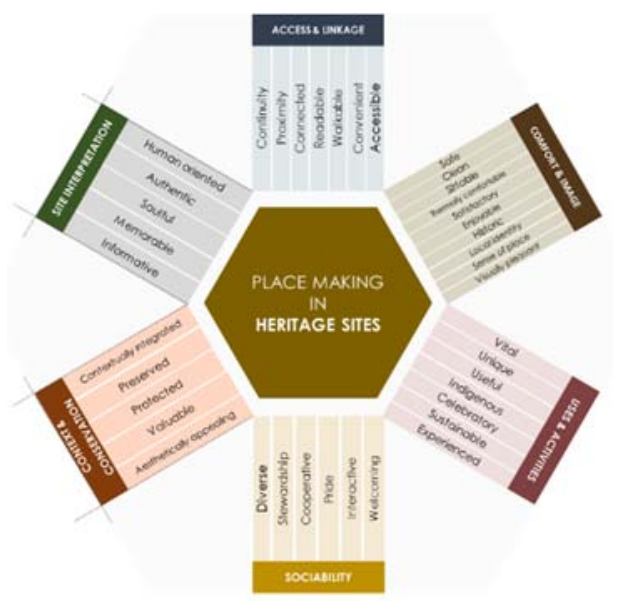

Figure 2: The proposed model [16]. 


\subsection{Model structure}

Referring to the previous discussion, we can configure the new formulated model after adjusting the original one, where the new domains are revealed. Although the demonstrated diagram shows the attributes and their aspects clearly, there is a critical issue that should be addressed where the configuration of the wheel model does not represent the intersected relationships among the indicators and aspects of the Placemaking model. It is represented as layers and sectors which make it inflexible for analysis. However, defining the aspects and understating them deeply; it shows that measuring the success of those aspects can be indicated through several indicators that contribute to the main aspects [16]. By considering this, the researchers developed a new morphology for the model structure to demonstrate the maximum interaction among the proposed qualities by representing it into a matrix form to be able to intersect and highlight common features and relationships within different levels.

\subsubsection{Components hierarchy description}

The key attributes or domains refer to the border strategy dimension of the project development that should be addressed. Each one consists of several aspects or principles which they encompass both tangible and intangible qualities concerned to make the place livable, soulful, and authentic. While this tends to look theoretical, bridging it to practice requires measured indicators to assess aspects' achievability within a place. Consequently, including this level is beneficial to the model's credibility in order to indicate the success level when implementing aspects. These components contribute to building the desired model proposed by the researchers (Fig. 3).

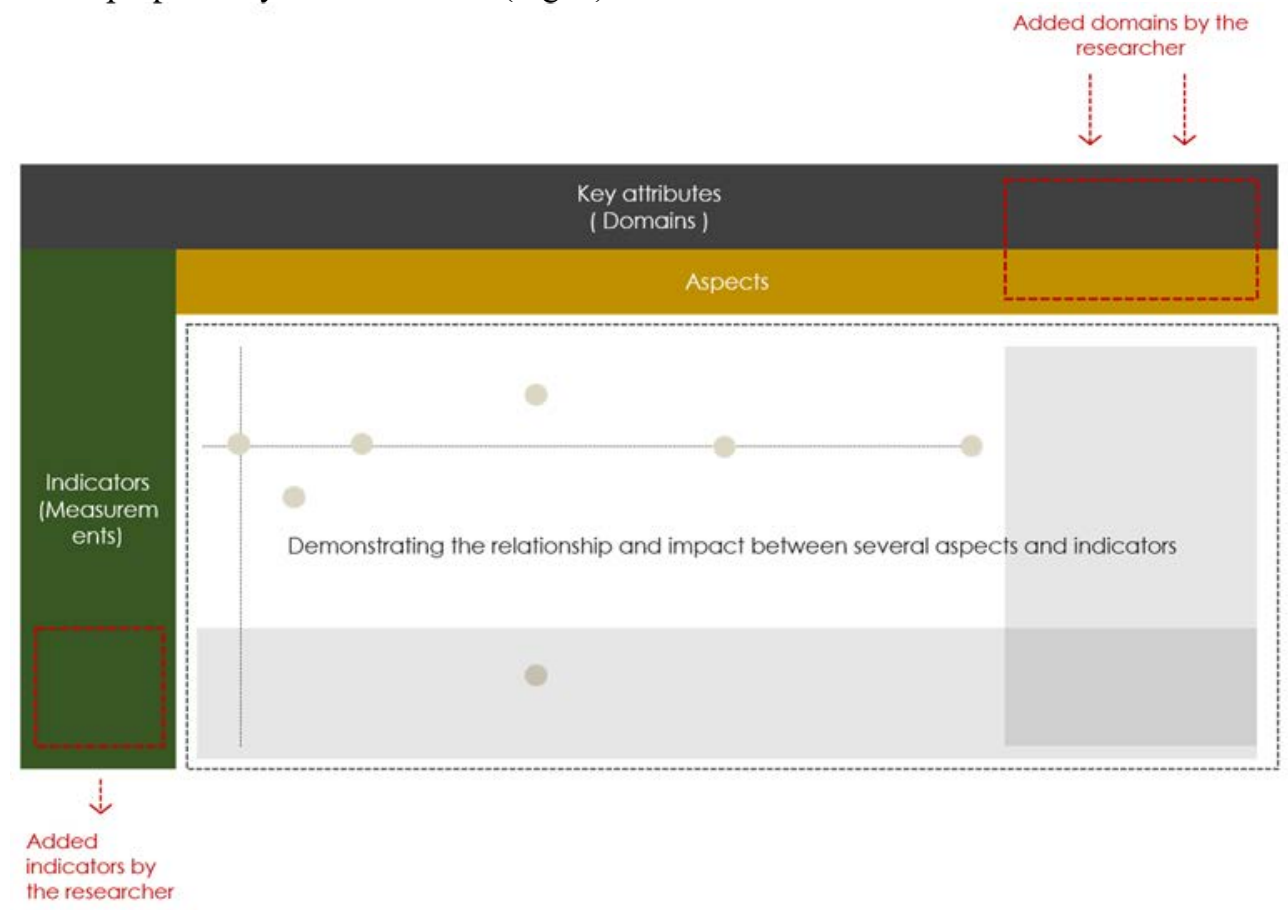

Figure 3: The proposed placemaking model structural diagram that illustrates the intersection between aspects and indicators [16]. 
Table 1: The adapted model formulated into the new model structure (matrix). (Source: Authors.)

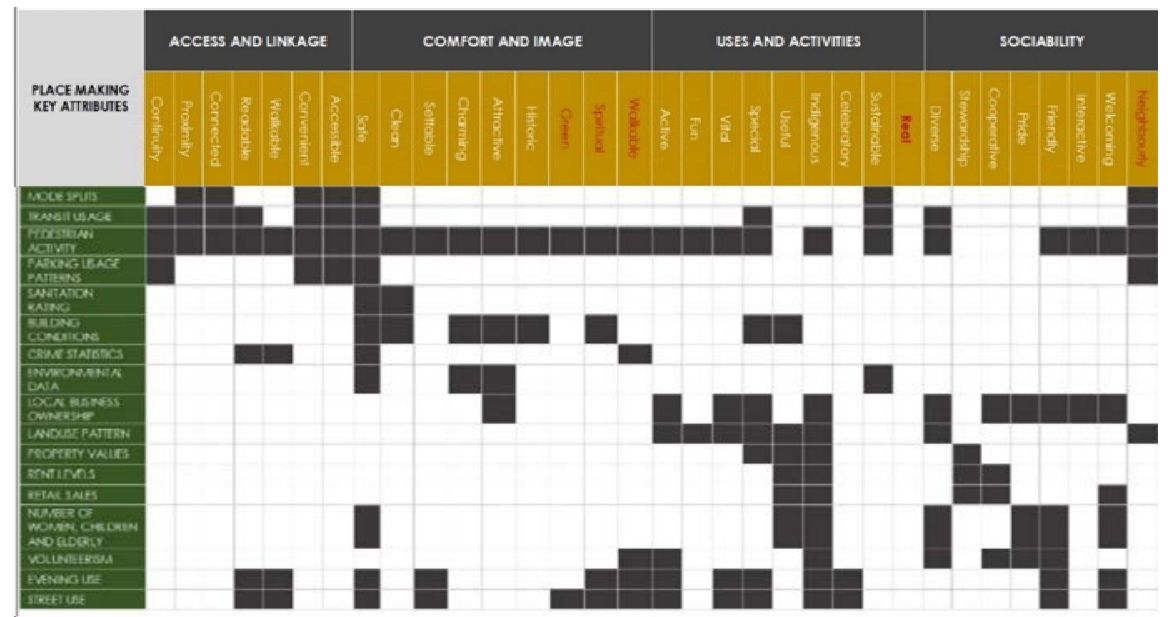

The proposed model tried to compensate for the shortages in which the previous attempts did not and exhibit the relationship between the new and original aspects as well as indicators. The new model is developed as a classified matrix (Fig. 3). The matrix format does help to represent the intersections and relationships among the horizontal and vertical axis. This will give an in-depth analysis of the aspects and their indicators in order to use them during the design and planning process. In the new structure; the key domains will be the horizontal header axis of the table representing the higher umbrella, while the aspects are subdivisions of them referring to the strong relationship between them; considering the highlighted new added domains/aspects later to differentiate between them (Table 1). On the other side, the vertical axis represents the indicators or measurements required to assess the aspects through them. Additionally, it does reveal the newly added indicators by the researchers. To end with the body part, which represents the interactive space (the intersection or impact of aspects with several indicators) which represented in the intersected circles within the middle space.

\subsubsection{Implementing the new structure on the developed proposal}

The adaptation of the original model on the new structure has remarkably revealed the interaction between indicators and aspects where it was not clear before (Table 1). The highlighted red aspects refer to the replaced aspects, as described earlier. Upon that, the researchers implemented the new proposal on the new structure representing the new aspects and domains, which are highlighted by light yellow, also, to new indicators represented in light green.

While the middle body of the matrix exhibits the impact between each aspect and a related indicator based on the in-depth understanding of the aspects and key attributes were discussed earlier; the research could predict the interrelationship among various measurements and aspects, therefore, it is filled accordingly. In that sense, it is essential to verify the new qualities and its validity and impact concerning urban heritage sites and cultural tourism. To validate the model credibility and practicality along with its aim and objectives; another critical step is to identify the importance of each aspect under each domain to configure the essential qualities that should be implemented in heritage and cultural place primarily than any other. 


\section{MODEL VERIFICATION AND WEIGHING}

The verification of the adapted model is required to ensure applicability and credibility. This action to be also followed by weighing the importance of aspects between one and another. A designed questionnaire, as a practical tool, was chosen to be addressed for experts in relevant fields, including urban planners, city planners, urban designers, architects, transportation and planning, heritage specialist, and tourism officials. The questionnaire aimed to investigate the practical aspects, which contribute to making the public realm of a heritage site where interactions, meaning, experience, and relations are placed on strengthening the connection between people and urban heritage places they share.

\subsection{Questionnaire objectives and process}

The questionnaire aimed to test and develop the proposed model defining the effective aspects and prioritizing the importance among them. In order to verify the added aspects concerned with heritage contexts, moreover, to identify the importance level of each aspect from an expertise point of view. The questionnaire was designed as in scaling importance style for each aspect on a scale from 1 to 5 scale bar where five represents the highest and one the lowest, respectively (Fig. 4(a)). It consisted of six main categories according to the key domains of the designed model. The participants also had the option to suggest another aspects where they think it is essential to be considered in developing the heritage sites.

\subsection{Discussion and findings}

The questionnaire has received feedback from 40 participants from various specialties in the field. The concluded results show the majority is leaning toward the importance rate 4 and 5, while the minor aspects have a scale with low rate importance 1 to 2, leaving scale 3 is the middle score where it was selected in a reasonable average. Since that, the average mean results for each aspect under each category will be calculated to come up with the average rate, representing the differences rates among them. This process will help to classify the

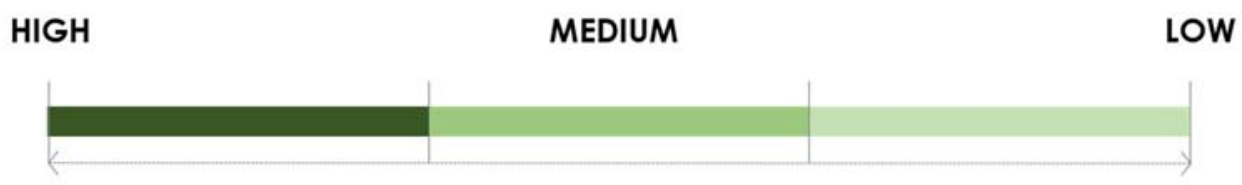

(a)

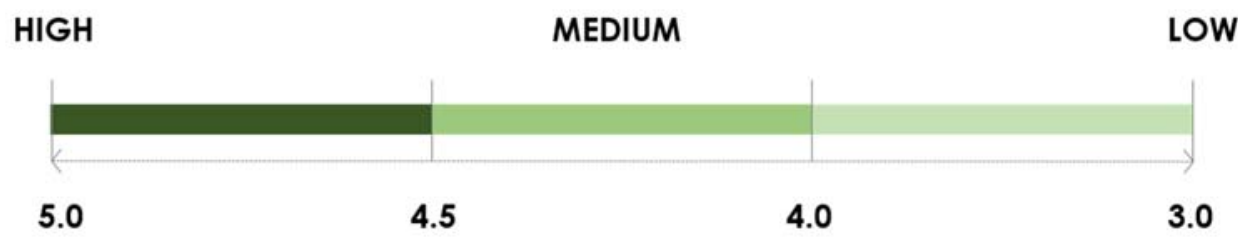

(b)

Figure 4: (a) The weighing method (high, medium, and low) classification; and (b) The updated scale bar for evaluating the average results for aspects [16]. 
importance rate for each domain group or exclude some when it is needed. Since all resulted average are scored 3 out of 5 , which means there was none such aspect was out of importance by participant's feedback leaving 3 is the minimum for each aspect, as such prioritizing these aspects we need to create a new range of scale 3-5 to assess the importance of each aspect related to all of the other aspects (Fig. 4(b)).

The following part will demonstrate the questionnaire findings categorized according to six sections which represent the key domains (attributes) of the model.

\subsubsection{Access and linkage attribute}

The resulted scores for access and linkage domain shows that for the site to be connected and walkable has scored as the highest priority to be implemented in heritage sites development. However, the accessible aspect has been scored by 4.45, which signifies its high importance as well. While the differences are quite remarkable in between medium to low priority where the medium scored for the aspects (readable, convenient and continuity) are scored between the range 4.18 to 4.225 which are all quite close to each other compared to the aspect (proximity) which scored 3.6; considered to be the lowest priority (Fig. 5(a)). While the other bar chart diagram represents the average results compared between the aspects shaded per importance; reflecting the key legend, which was described earlier (Fig. 5(b)).

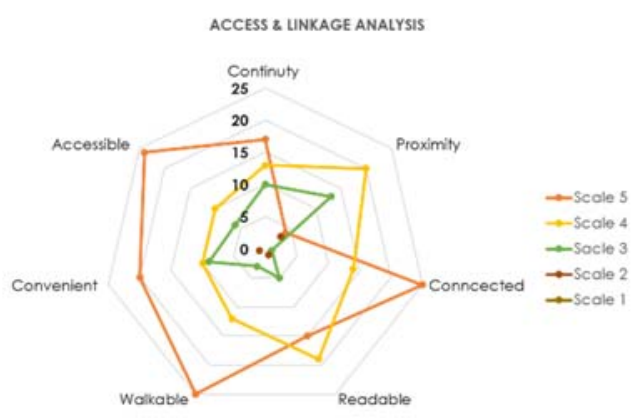

(a)

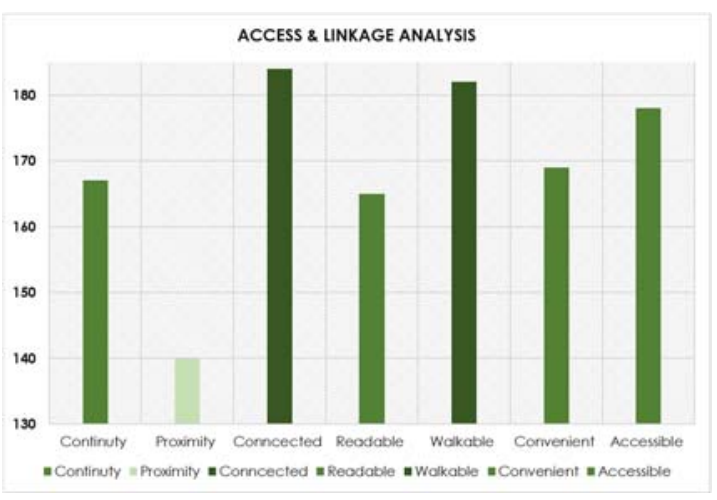

(b)

Figure 5: (a) Access and linkage domain; and (b) Average values of access and linkage domain [16]. 


\subsubsection{Comfort and image attribute}

For the domain comfort and image, the results are quite different from the previous domain. The majority of aspects have ranged as medium importance from 4.33 to 4.17 which are proximate to each other, while the aspect (safe) is signified as the highest importance among them all, it has scored (4.8); this indicates the importance to achieve safety and security for visitors and tourist at the first place while developing heritage sites and bringing people to the place. On the other side, the aspects which contribute to achieving visitors' comfort (sittable and thermal comfort) have scored as the lowest among them was 3.6-3.7. This indicates that these dimensions come right after achieving the essential aspects that represent the heritage sites as it should be like achieving clean, historical, local identity, enjoyment, visually attractive elements, and sense of place (Fig. 6(a)). On the other hand, the bar chart does represent the average rate results for the aspects concerning each other (Fig. 6(b)).

\subsubsection{Uses and activity attribute}

Uses and activities domain in general exhibits the lowest resulted priority, among others. Where the received scores have varied between only medium to low, referring to the key introduced scale. While it represents that (sustainable and experienced) aspects were the

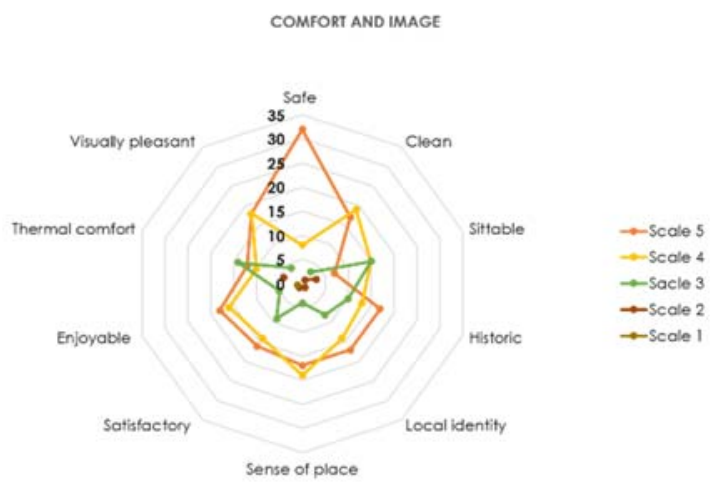

(a)

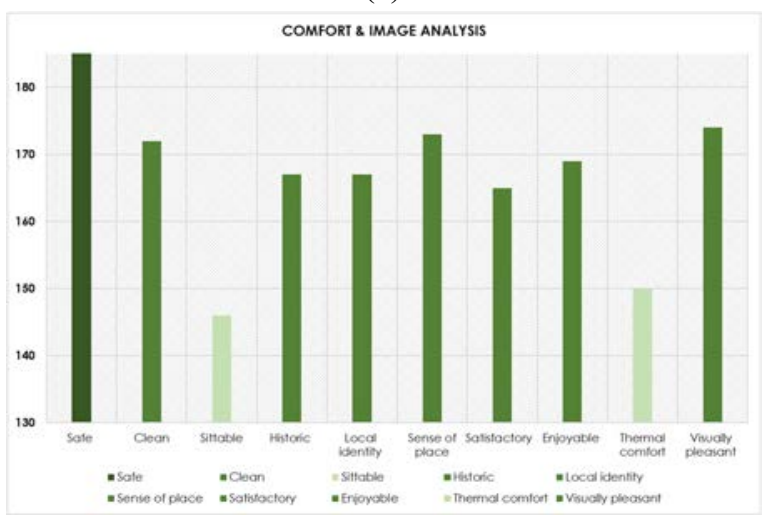

(b)

Figure 6: (a) Comfort and image domain; and (b) Average values of comfort and image domain [16]. 
highest medium scored. The aspect unique tends to be medium importance as well as it isscored 3.97 whereas the lowest category (useful, indigenous, celebratory and vital) are prioritized from high to low, respectively (Fig. 7(a)), while the bar chart demonstrates the average rate amongst them (Fig. 7(b)).

\subsubsection{Sociability attribute}

We can identify the most significant aspect among this domain, which is (welcoming); this indicates that people first judge the place entering planning strategy and design and how welcoming it is, which contributes to the level of their satisfaction of visiting the heritage site. To be followed with other supportive aspects that achieve sociability and cooperation between people in the place. Where this also reflects in the scored results as (interactive, pride and diverse) have medium-scale ranged from 4.25 to 4.0 where they are closely rated; this signifies the relationship between the intangible aspects that is concerned with human emotions, feelings, and perceptions of the place which are almost equivalent in priority.

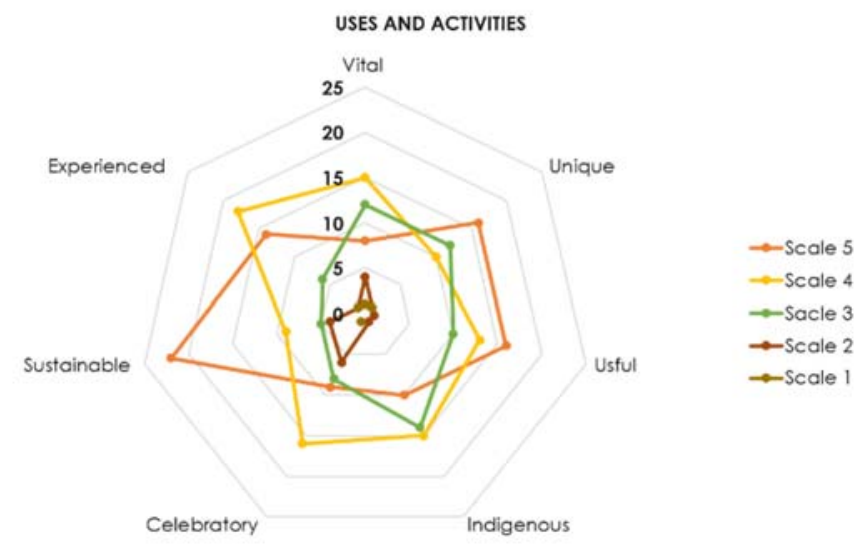

(a)

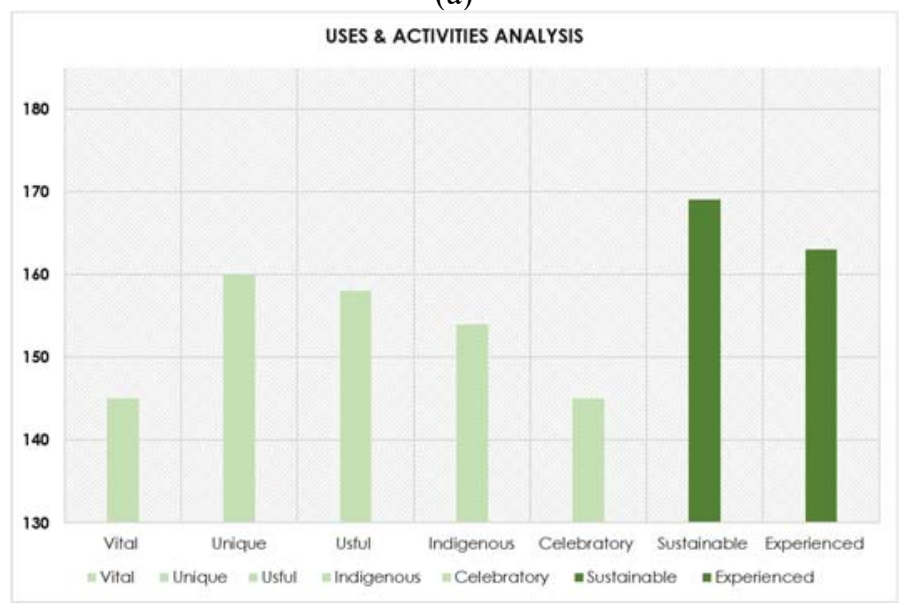

(b)

Figure 7: (a) Uses and activities; and (b) Average values of uses and activities domain [16]. 
However, results show that participants have the lowest priority goes to stewardship and cooperative; this may indicate that these aspects can be achieved directly by managing others (Fig. 8(a)), while Fig. 8(b) reveals the common findings of the related aspects of the sociability domains.

\subsubsection{Context and conservation attribute}

The results of the context and conservation domain prove its significance and priority among other domains where it is remarkably seen that the range of results from high to medium only, and none of them is considered a low priority. The maintenance aspect has significantly rated as the highest, which was scored by 4.7 ; this indicates that maintenance in heritage sites is essential to be achieved in consideration of preserving and protecting the heritage assets. Other aspects have been scored as medium importance as they are scaled from 4.4 for valuable aspect, 4.35 aesthetics and finally 4.225 for urban context integration (Fig. 9(a)). The results show the high importance of all aspects relevant to this domain from the highest to lowest (Fig. 9(b)). Identifying the values of heritage assets, both tangible and intangible dimensions, are essential dimensions to indicate success in placemaking heritage, strengthen that by also paying attention to aesthetics factors that contribute to achieving excellent reputation, attractiveness, and satisfaction. One other, integrating urban context around

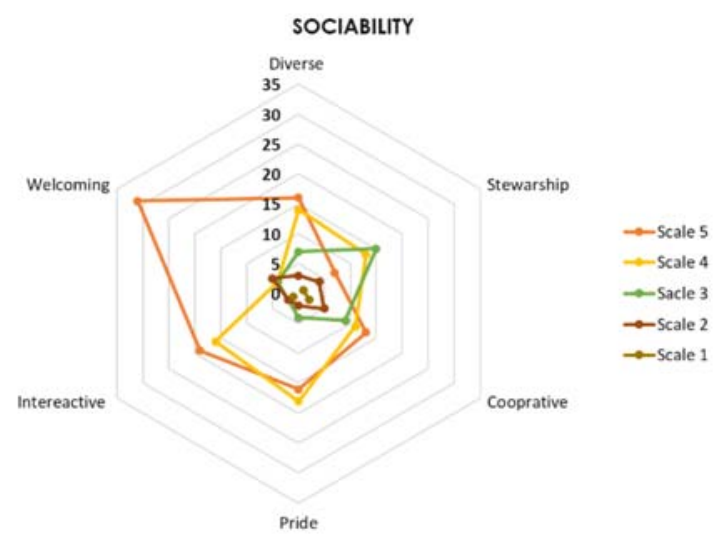

(a)

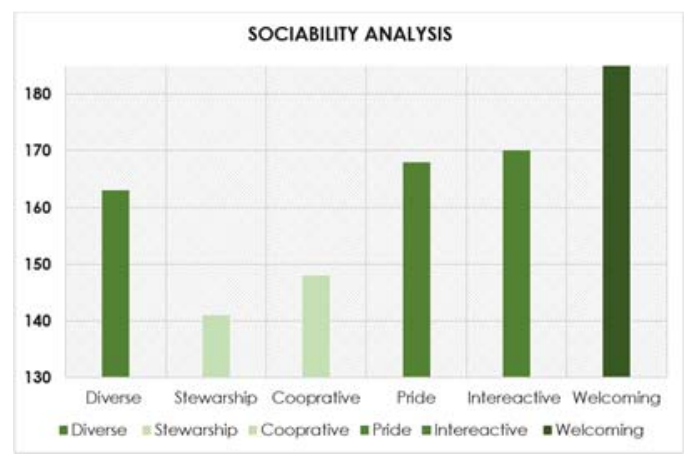

(b)

Figure 8: (a) Sociability domain [15]; and (b) Average values of sociability domain [16]. 


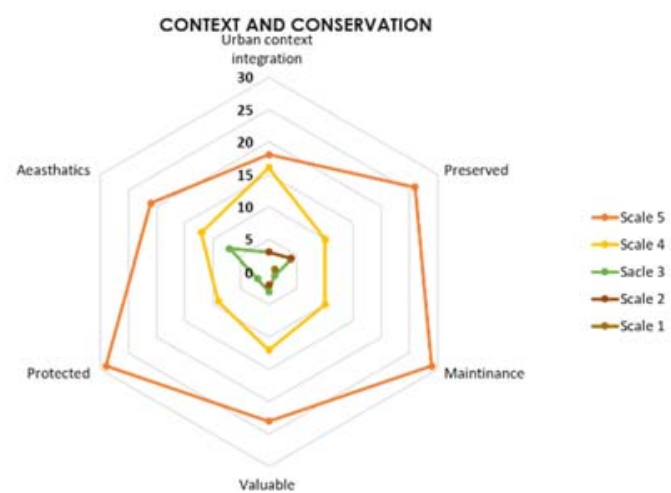

(a)

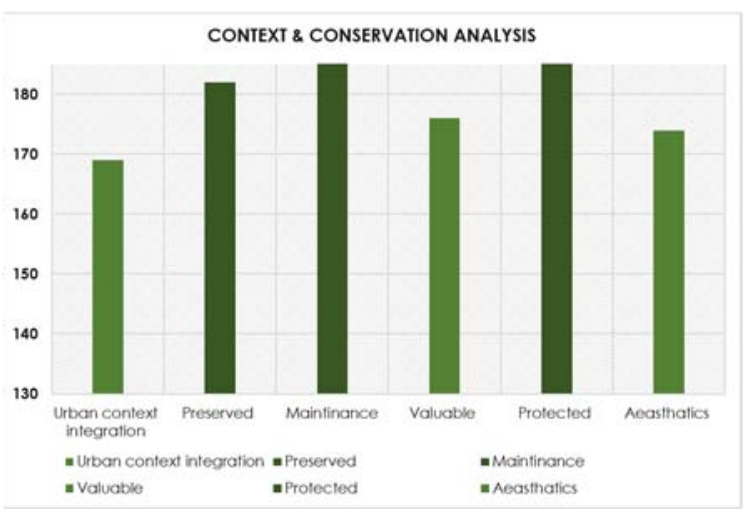

(b)

Figure 9: (a) Context and conservation findings [15]; and (b) Average values of context and conservation domain [16].

heritage in the development considering adjacent urban areas, can contribute to success for the area [9]. Furthermore, it reduces the pressure on heritage assets, especially within touristic seasons and increasing the capacity of visiting as well as, it contributes to preserving and elaborate local identity and belonging by developing neighboring areas as a valuable one.

\subsubsection{Site interpretation attribute}

The resulted scores for site interpretation domain are smoothly rose up from 3.8 to 4.52 . The highest score was for the aspect (informative), while the lowest was for (soulful) aspect. This indicates that participants promote cultural awareness and knowledge for the first level in order to achieve a good reputation, identity, and engaging storytelling within the provided activities. Besides, fulfilling a memorable experience that is humanized and authentic contribute to enrich the interpretation of the place and accomplish soulfulness accordingly (Fig. 10(a)). The bar chart shows the average rate results amongst all aspects (Fig. 10(b)).

The outcome results show the significance and importance of integrated domains and aspects incorporated in forming the model of heritage placemaking regardless of the inner prioritization among one another. Therefore, the added components were automatically 


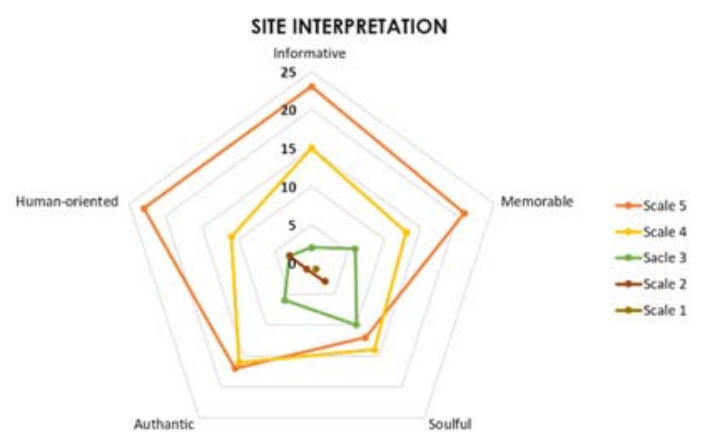

(a)

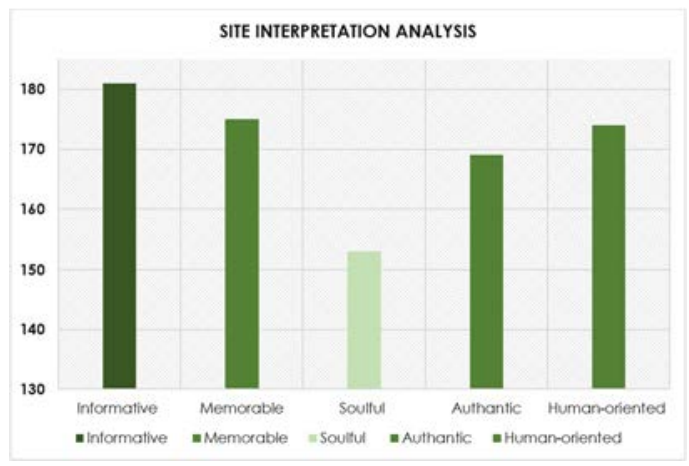

(b)

Figure 10: (a) Site interpretation domain findings; and (b) Average values of site interpretation domain [16].

verified to be part of the model. While there is a variation of importance levels were evident in some domains. Therefore, these results contribute to modify the proposed model as per importance level in order to benefit the end-user to adapt the model while in the design process, assessing an introduced one or helps to make decisions by stakeholders.

\section{THE DEVELOPED PLACEMAKING MODEL FOR SENSITIVE HERITAGE SITES}

The proposed placemaking model for heritage sites has been built in light of the traditional placemaking model; six major pillars are required to deliver an integral placemaking on heritage sites and attract cultural tourism. Within those pillars, a set of qualities that contribute to achieving them, where they verified and weighted by the importance which represented along with its relevant indicator. This was followed by implementing those findings on the final matrix to be linked with this representation and arranged according to priority among aspects of each domain separately (Fig. 11).

A developed matrix as final representation was extracted from the previous findings and results. As shown, the main domain has been color-coded while each domain's aspects have been arranged according to resulted priorities, where the intersections between aspects and indicators have been updated accordingly. The outcome model represents results adaptation on its final representation where it is classified between one to another (Table 2). 


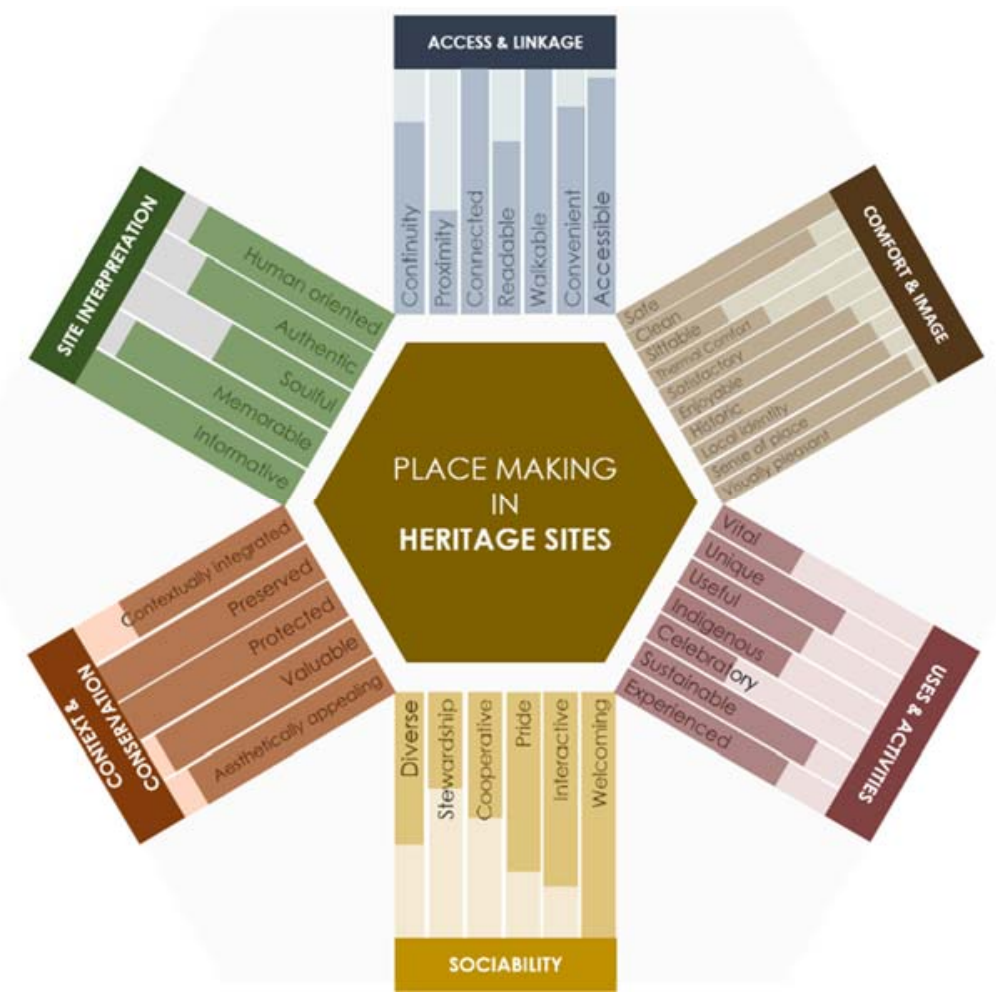

Figure 11: The updated model [16].

Table 2: Proposed matrix integral placemaking model for heritage sites. (Source: Authors.)

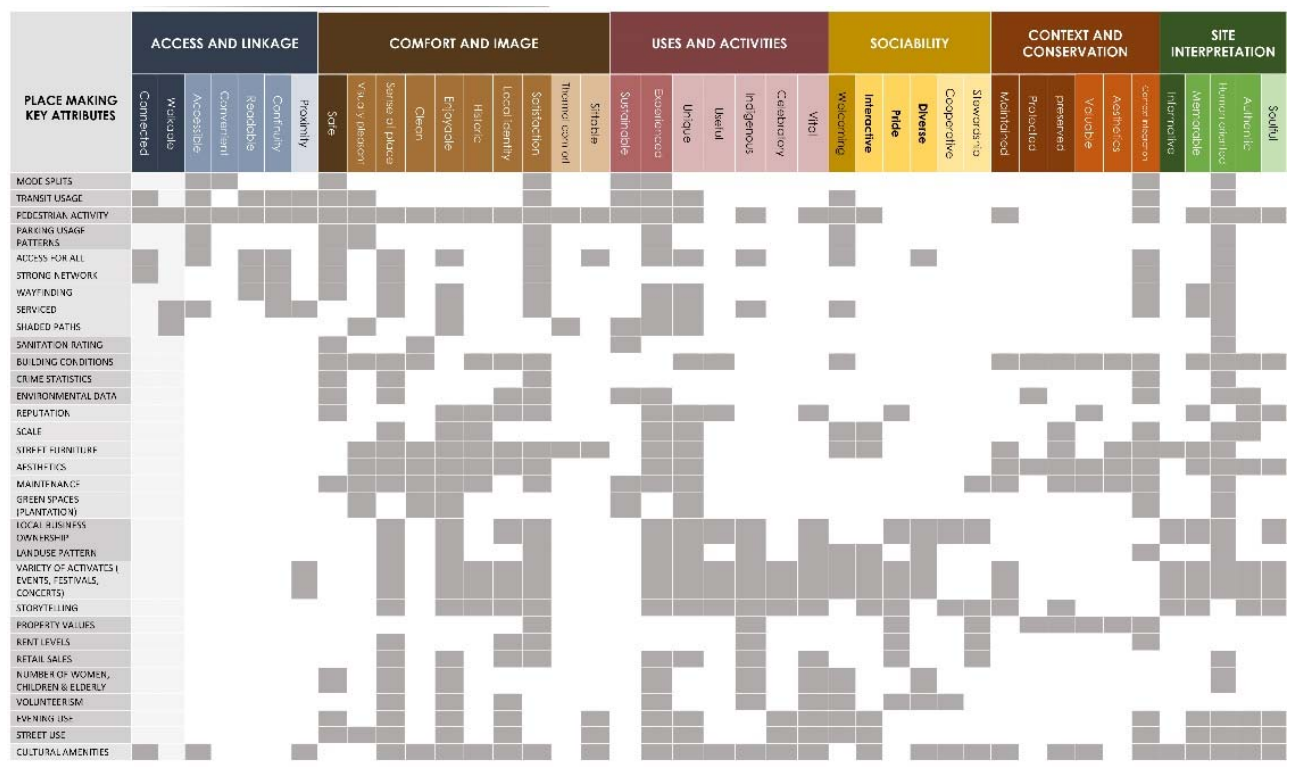


The outcome of this research demonstrated above contributes to various benefits for the research community, planners and urban designers. The model can be used variously, firstly, to be used in the initial planning process as a design criteria tool in developing the design in relevance to the attributes/aspects priority. Secondly, the developed model benefits the decision-makers and stakeholder who are in charge to develop heritage sites and promote tourism to take the right decision by referring to it; in which the model can help to assess the crucial aspects which need a priority in investments and taking actions. Finally, the model can also be used as an assessment tool in the case of evaluating proposed projects in order to identify the strengths and shortage aspects that occur in the proposed design.

\section{CONCLUSION}

The key findings of this research outlined the placemaking approaches and how the researchers developed the taxonomy of the main aspects contribute in heritage sites placemaking process. The conducted questionnaire, through the model building process, has strengthened the model credibility, contributed to generating the outcome, which is the subject of this research. The discussion has been elaborated to a certain extent to include the potential, benefits, usage opportunity associated with the developed placemaking model of heritage and cultural sites. Finally, it is concluded by potential reflection on the design process workflow to illustrate the researchers' aim in bridging the theory and practice through the developed matrix.

\section{REFERENCES}

[1] Saudi Vision 2030, www.vision2030.gov.sa/ar.

[2] Pendlebury, J., Preservation in the Age of Consensus, Routledge: London, 2009.

[3] Pendlebury, J., Conservation and regeneration: Complementary or conflicting processes? The case of Grainger Town, Newcastle upon Tyne. Planning Practice \& Research, 17(2), pp. 145-158, 2002.

[4] UNESCO, Recommendations on the Historic Urban Landscape, UNESCO: Paris, p. 8, 2011.

[5] Elbelkasy, M.I., AlSalafi, J.M. \& Mahmoud, M.F., Re-use of historical buildings in the holy city of Makkah: Case study selection guidelines. WIT Transactions on The Built Environment, vol. 153, WIT Press: Southampton and Boston, pp. 63-74, 2015.

[6] Duxbury, N., Hosagrahar, J. \& Pascual, J., Why must culture be at the heart of sustainable urban development? Culture, 21, p. 36, 2016. www.agenda21 culture.net.

[7] Department of the Environment, Planning Policy Division, Living Places: An Urban Stewardship and Design Guide for Northern Ireland, 2014.

[8] Victoria State Government, DELWP Services. planning.vic.gov.au.

[9] Svirplys, L., Planning Considerations for the Implications of Local Government Restructuring on the Sense of Place in a Rural Community, University of Guelph, 1999.

[10] Bandrin, F. \& Oers, R.V., The Historic Urban Landscape, Managing Heritage in Urban Century, Wiley-Blackwell, 2012.

[11] Tuan, Y.F., Space and Place: The Perspective of Experience, University of Minnesota Press: Minneapolis, 2018.

[12] Lynch, K., The Image of the City, MIT Press: Cambridge, MA, 1960.

[13] Ouf, A.M., Authenticity and the sense of place in urban design. Journal of Urban Design, 6, pp. 3-4, 2001.

[14] PPS, What is Placemaking, What if We Build Cities Around Places? Project for Public Spaces Inc., 2018. www.pps.org. 
338 Structural Studies, Repairs and Maintenance of Heritage Architecture XVI

[15] Alawi, G., Jamjoum, H. \& Samir, H., Enhancing the cultural tourism experience: The case of historical Old Jeddah. WIT Transactions on The Built Environment, vol. 177, WIT Press: Southampton and Boston, pp. 39-50, 2018.

[16] Samargandi, S., Integral placemaking in sensitive heritage sites for successful cultural tourism. Master's thesis, Effat University, Jeddah, KSA, 2019. 